\title{
Distributed Cloud Operating System (DiCOS) Development at Academia Sinica
}

\section{Eric Yen}

Academia Sinica Grid Computing Centre (ASGC)

\#128, Sec 2, Academic Rd., Nankang, Taipei, Taiwan, 11529

E-mail: Eric.Yenetwgrid.org

\section{Felix Lee}

Academia Sinica Grid Computing Centre (ASGC)

\#128, Sec 2, Academic Rd., Nankang, Taipei, Taiwan, 11529

E-mail: felixatworid.org

\section{Weijen Chang}

Academia Sinica Grid Computing Centre (ASGC)

\#128, Sec 2, Academic Rd., Nankang, Taipei, Taiwan, 11529

E-mail: weijen.changatworid.org

\section{Cheng-Hsi Chao}

Academia Sinica Grid Computing Centre (ASGC)

\#128, Sec 2, Academic Rd., Nankang, Taipei, Taiwan, 11529

E-mail: chenghsi.chaoltwgrid.org

\section{Asa Hsu}

Academia Sinica Grid Computing Centre (ASGC)

\#128, Sec 2, Academic Rd., Nankang, Taipei, Taiwan, 11529

E-mail: asa.hsultworid.org

\section{Ming-Jyuan Yang}

Academia Sinica Grid Computing Centre (ASGC)

\#128, Sec 2, Academic Rd., Nankang, Taipei, Taiwan, 11529

E-mail: mandy.yangetworid.org

\section{Zong-Tsung Wu}

Academia Sinica Grid Computing Centre (ASGC)

\#128, Sec 2, Academic Rd., Nankang, Taipei, Taiwan, 11529

E-mail: tsung-hsunetwarid.org 


\begin{abstract}
Distributed Cloud Operating System (DiCOS) is designed to support large and long-tail data analysis efficiently in all disciplines by federating distributed Cloud resources. The distributed infrastructure established by DiCOS federates small data centers and provides low latency access and high performance applications to local users. Job is submitted locally and run globally while reducing the number of data transmitted across resource centers. DiCOS could provide more than $400 \mathrm{~K}$ CPU-days computing power to support several application domains with 6 resource sites in Taiwan, US and CERN. DiCOS is becoming the new generation research infrastructure of Academia Sinica. In addition to high throughput, application efficiency is improved continuously by the optimization of workflow and system efficiency. The objectives and status of DiCOS, as well as the development strategy and plan for the second stage from 2016 are described in this study.
\end{abstract}

Keywords: Distributed Computing Infrastructure, Cloud, System Efficiency

International Symposium on Grids and Clouds 2016

13-18 March 2016

Academia Sinica, Taipei, Taiwan 


\section{DiCOS Introduction}

Distributed Cloud Operating System (DiCOS) is designed to support large and long-tail data analysis efficiently in all disciplines by federating distributed Cloud resources. The core technologies are based on ATLAS computing from WLCG to leverage the stable evolution of distributed computing technologies with heavy usage. ASGC joins the technology development at CERN focusing on applications requirements from non-HEP user communities, such as the web user interface, MPI support, automatic deployment, container integration, single sign-on, core components generalization, etc. System efficiency is the primary target when DiCOS turns into production in 2014. Efficiency comes from actively reacting upon user experiences as well as the continuous improvements of power, thermal and system efficiency.

Evolution of efficient data analysis from petabyte scale towards exabyte scale is the most demanding capability in the next decade for not just the academic activities, but also applications of industry and people's daily services. Conventional centralized Cloud center suffers from limited throughput and access latency issues while the cloud services scale up. Distributed infrastructure has been proved to be effective for big data processing $\&$ analysis, and large-scale international collaborations by academic projects such as WLCG[1], LIGO[2], ELIXIR[3], climateprediction.net[4], etc. Naturally, the virtual world will be consisted of grids of clouds to serve the computing needs of enterprises and individuals with reduced latency.

DiCOS aims to provide a shared distributed computing infrastructure for various applications of research, education and industrial fields covering applications of big data, long tail and small data. The distributed infrastructure established by DiCOS federates small data centers and provides microsecond latency access and high performance applications to local users. Most jobs could be finished at the nearest site with the advantages of latency and less data transmission. Job could be running at the best available site and data is moved transparently when remote resource is required. Combining DiCOS with energy efficient and noise-free hardware, the single rack data center can be setup as a building block of cloud resources for various scales such as a lab, an institute or a whole campus. Job is submitted locally and run globally while reducing the number of data transmitted across resource centers. Distributed job management $[5,6,7,8]$ and distributed data management $[9,10,11]$ are the foremost core technologies we participated in the past ten years.

The first version DiCOS has been online and supporting Alpha Magnetic Spectrometer (AMS-02) experiment data analysis since 2013. DiCOS keeps improving based on users feedback and evolved technologies. With capabilities of automatic deployment and software distribution management, DiCOS could be setup at collaboration sites easily and consistently. For the moment, DiCOS has been supporting applications on high energy physics, bioinformatics, earth science, environmental changes, financial engineering and physics. Besides the installation at Institute of Physics, Institute of Earth Science, Research Center of Environmental Changes and ASGC in Academia Sinica, DiCOS had been deployed at National 
Central University, National Dong Hua University, National Center of High-performance Computing, University of Chicago and CERN.

Subjects of next phase DiCOS development from 2016 would include: 1) system efficiency enhancement; 2) supporting more new applications such as bioinformatics and extending DiCOS resource network; 3) incorporating new energy-aware workload management framework such as smart power management and live VM migration; 4) incorporating container technology.

\section{Development Approach}

DiCOS is the first and largest production distributed cloud system supporting multiple big data academic applications in Taiwan. DiCOS keeps enhanced according to more user experiences and by deploying at more collaboration sites such as University of Chicago, CERN and collaborative institutes outside of Academia Sinica. For the most efficient IT system, it is only achievable by coping with power, energy and operation efficiency at the same time. The distributed infrastructure made by SRDC is the best solution to circumvent latency issues of a large application or applications with users dispersed in different places around the world.

Based on user experiences and application requirements of scientific communities, the core components of DiCOS keep improving, covering distributed job management, distributed data management, storage system, Web user interface, security, monitoring and control, and system efficiency. Architecture and software stack of DiCOS is depicted as Figure 2. Effective use of distributed resources for large volume of jobs and minimize the job latency and CPU waiting time is the first goal of the DiCOS Distributed Job Management component. Job might be running at the best available site and data is moved transparently across sites for some scenarios. Jobs are taken care by the pilot factory by pulling jobs from the available computing nodes. Computing nodes is dynamically provisioned with automatic deployment of required application environment. Application workflow is customized according to the computing model, apart from the generic web user interface. Data availability decides how the jobs would be executed and intelligent staging ensures the minimization of data transmission overhead. Integration of the system information system is essential for the smart wrapper (implemented by the pilot factory) to find the best resource for jobs and also re-submit failed jobs automatically. Until there is no jobs in the queue pilot then ceases to exit. In addition to customization and optimization according to local users requirements, our own development are focusing on following components for now: Web User Interface, Application Program Interface, parallel computing, automatic installation, assess management system, and private cloud integration etc.

In coping with various computing models from different disciplines of varied virtual organization (VO), DiCOS made some changes to the middleware. Foremost examples are summarized as follows. 
1. Database for both Distributed Job Management and Distributed Data Management were changed to be MariaDB from Oracle. To reduce the setup and operational cost and to facilitate the sharing of DiCOS middleware, DiCOS sticks to open collaboration and has all components open sourced. High availability and load sharing architecture are the necessary model applied to the database server.

2. Distributed Job Management: To simplify job submission workflow, DiCOS supports dynamic brokering to arrange jobs to the best available sites according to users requirements without user's knowledge of the job queue configuration and status. Information system integrating configuration and status information of cloud resources, services, sites and topology is applied. For supporting multiple VOs, customized plugins were developed. Configurations for broker, job queues, dispatcher, and pilot factory were all revised accordingly. Rucio is used as the data management system for DiCOS.

3. Distributed Data Management: RUCIO provides the distributed data services for DiCOS, such as data discovery, data transmission, data deletion, data consistency and computing model enforcement. Integration of DJM and DDM for applications other than ATALS has to be modified accordingly. Web user interface for DDM is developed to support easy drag-and-drop operation to data at DDM in the hierarchical data directory view of the files. (figure $\mathrm{X}$ ) Xrootd protocol for data transfer between storage elements especially for the backend EOS storage system is also developed. Ceph has also been integrated as a backend storage pools to support block and object storage services for other DiCOS components. Benefited from WLCG, File Transfer Services are used to support sophisticated scheduled transfers by multiple protocols between storage endpoints.

4. DiCOSBox: Based on the collaboration with CERNBox development, DiCOSBox is used to integrate synchronization and sharing with user workflow and provide direct access to user data with multiple protocols anywhere in the world. The current version is to use EOS as the backend virtually unlimited cloud storage.

5. Cloud: Virtual machines (VM) and hypervisors are managed by OpenStack and VM could be created on-demand whenever there is any job coming. In addition to bare metal and VM resources are included, container inclusion is now under development. MPI applications over the cloud is the next top focus for DiCOS.

6. Application Programming Interface (API): RESTful API of DJM and DDM have been developed to support application integration and tools/services development by 3rd party. Job submission, cancellation, job information and full set of distributed data management have been in place. In addition, authentication, VOMS information and proxy management will be online in next year. 
7. Web User Interface and Single Sign-On: Lightweight Directory Access Protocol (LDAP) is used to support account and certificate management for DiCOS. There is no need of multiple logins to access to DJM or DDM in DiCOS. From DiCOS Web UI, the processes from proxy initiation using user certificate to the job submission and data management could all be achieved at the DiCOS Web UI. Furthermore, tailored web interfaces were carried out according to the computing model and design of user communities. From DiCOS WebUI, user could user ROOT for data analysis and visualization on-the-fly. Moreover, app for both Android and iOS are also released to support mobile usage for job submission and status check.

8. Monitoring and Control: Based on minute-level resolution monitoring of system status, resource usage statistics in worknode and in job are carried out all the time. For the goal of system efficiency optimization, anomaly such as failed job with large quantity of CPU usage, high failure rates of a worknode, idle CPU time of a worknode in contrast to number of waiting jobs at queues, as well as high I/O wait ratio and memory utilization status etc., are all analyzed to discover the patterns and metrics of identification. The ultimate goal is to make the anomaly detection and resolution process to be as smart as possible which requires deeper analysis and machine learning technologies.

Revision to the core components were feedback to WLCG and maintained the applicability to the latest version. Release management of DiCOS and quality assurance are supported by DevOps framework such as Jenkins, GitLab and Elasticsearch, etc.

\section{DiCOS Status and Achievement}

AMS is the first and largest user community of DiCOS since 2013. VMs for AMS jobs will be created when the pilot get the waiting jobs. AMS jobs were executed at several clusters at ASGC and also in the resources at National Central University which is around 100KM away from ASGC. The CPU usage of AMS jobs have a steep jump from 150K CPU-days in 2014 to more than 400K CPU-days in 2015. The usage is 2016 would be very likely as the scale of 2015. To further support data access to AMS users who are mostly at CERN, a DiCOS cluster at CERN was setup and in operation in 2016. The CPU usage of AMS at DiCOS in comparison with the CPU utilization of ATALS is depicted as Figure 3.1.

From 2015, DiCOS has been supporting bioinformatics applications on RNA-sequence data analysis for cancer studies in collaboration with University of Chicago and Northwestern University. Around 1,000 public datasets from GEUVADIS were processed by DiCOS at the first stage according to the workflow designed by University of Chicago. Jobs are wrapped up as a container and submitted to DiCOS. Input data were registered at DDM in DiCOS so there is no need to download from the original source again when the data has to be reprocessed. Output of the analysis is registered in DDM as well and shared with University of Chicago. Northwestern University supports the provisioning and configuration of software-defined 
network for the bioinformatics data transmission between Taipei and Chicago. One more DiCOS resource has been established at another collaboration of the National Center for Highperformance Computing (NCHC) in Taiwan. DiCOS is going to be setup at University of Chicago from 2016. Architecture of support RNA-Seq data analysis pipelines on DiCOS could be found at Figure 3.2.

Financial engineering is the most recent application of DiCOS in 2016. A large scale of portfolio simulation jobs will be executed. It is estimated to have $100 \mathrm{~K}$ CPU-hours usage in 2016. However, the input and output data scale is much less in comparison with AMS.

While DiCOS is extending its application domains, system efficiency optimization is the primary guiding principle on the development and operation. For example, to maximize the CPU utilization, whenever job anomaly pattern is identified, the cases will be filtered out for further analysis from the daily monitoring. For the moment, jobs with abnormal running time and duration time ratio, long idle time, unusual stage-in rate or data size, etc. have been singled out. Patterns and root causes of failed jobs, especially those failure jobs consuming a lot of CPU time, will be analyzed to minimize the CPU usage of jobs which are not possible to be finished successfully in the end. Performance of data stage in and data transmission inside the data center and between remote DiCOS sites will be improved. Reducing CPU idle time during the transition between single-core jobs and multi-core jobs in a computing node is also our focus. Summarized statistics on single-core jobs and multi-core jobs respectively of WLCG Tier-1 resources at ASGC is shown in Figure 3.3. The same statistics of WLCG Tier-2 resources at ASGC is shown in Figure 3.4.

DiCOS established the distributed computing infrastructure for scientific applications in Taiwan. Development of DiCOS is based on application experiences, user requirements and emerging technology, while system efficiency standing as the most essential rule. DiCOS keeps improving its easy access to the distributed resources and easy integration with various computing models while the DiCOS network is extending to wider user communities and collaboration sites. It is not possible to move forward along the path we proposed without the foundation of those works we achieved and the benefits of international collaboration.

\section{Future Perspective and Summary}

DiCOS is becoming the new generation research infrastructure of Academia Sinica and collaboration institutes. In addition to high throughput, application efficiency is improved by the optimization of workflow and system efficiency. Sharing of common data, tools, services and knowledge is straightforward, which is especially practical for collaborations with distributed partners around the world. Application is able to make use of the federated resources and benefit from the scalability and availability of DiCOS. Web User Interface could be customized for each Virtual Organization. RESTful API is provided for flexible integration with existing application framework or with 3rd party components. Data and resource protection could be achieved as a Private Cloud. Seamless integration with existing storage and file servers are also supported. 
In the second development stage from 2016, DiCOS will focus on the key areas such as: 1) integration with the Academia Sinica identity management system to further reduce the access barrier; 2) integrate Container-based framework for more flexible application workflow implementation and resource utilization; 3) buildup smarter efficiency improvement and optimization mechanism by incorporating machine learning technology; 4) providing more elastic MPI-based application support; 5) SDN integration for networking and data transmission efficiency; 6) federation of distributed DiCOS systems.

\section{References}

[1] Worldwide LHC Computing Grid (WLCG), http://wlcg.web.cern.ch

[2] Laser Interfermeter Gravitational-Wave Observatory (LIGO), http://ligo.org

[3] ELIXIR: A Distributed Infrastructure for Life-Science Information, https://www.elixir-europe.org

[4] climateprediction.net: the World's Largest Climate Modeling Experiment for the 21st Century, http://www.climateprediction.net

[5] F. Megino and J. Bejar et al on behalf of ATLAS Collaboration, PanDA: Exascale Federation of Resources for the ATLAS Experiment at the LHC, EPJ Web of Conferences, Vol. 108 (2016).

[6] T. Maeno and K. De et al, Evolution of the ATLAS PanDA Workload Management System for Exascale Computational Science, Journal of Physics: Conference Series, 513(3):032062, 2014.

[7] P. Nilsson and K. De et al, Extending ATLAS Computing to Commercial Clouds and Supercomputers, PoS(ISGC2014)034.

[8] V. Aulov and K. De et al, Workload Management Portal for High Energy Physics Applications and Compute Intensive Science, Procedia Computer Science, Vol. 66, pp.564-573, 2015.

[9] M. Barisits et al, on behalf of ATLAS collaboration, Resource Control in ATLAS Distributed Data Management: Rucio Accounting and Quotas, Journal of Physics: Conference Series, 664(6):062002, 2015 .

[10] V. Garonne, R. Vigne, G. Stewart, and A. Nairz, Rucio - The next generation of large scale distributed system for ATLAS Data Management, Journal of Physics Conference Series, 513(4):042021, June 2014.

[11] M. Lassnig and R. Vigne et al, Scalable and Fail-Safe Deployment of the ATLAS Distributed Data Management System Rucio, Journal of Physics: Conference Series, 664(6):62027, 2015. 


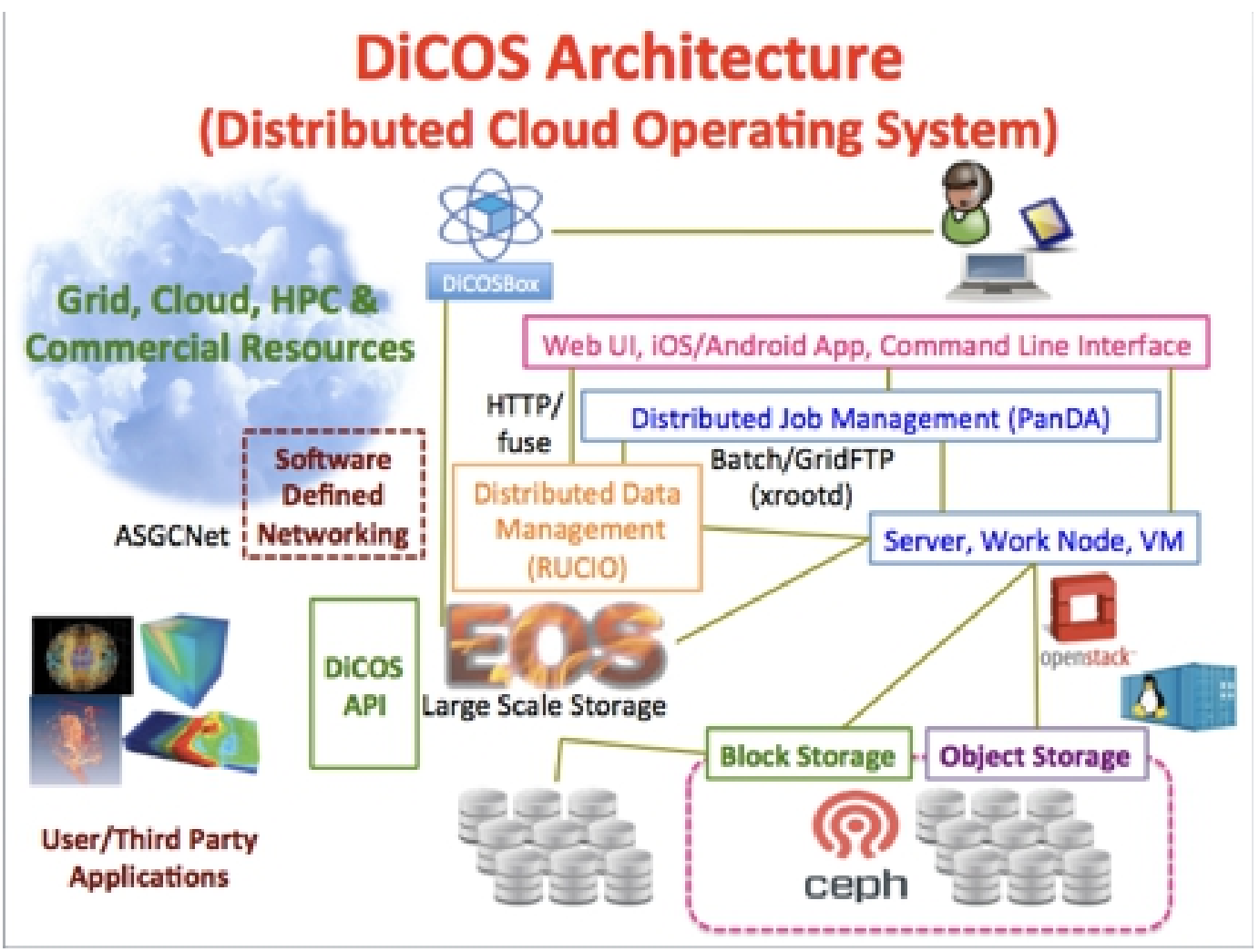

Figure 2.1. DiCOS logic architecture

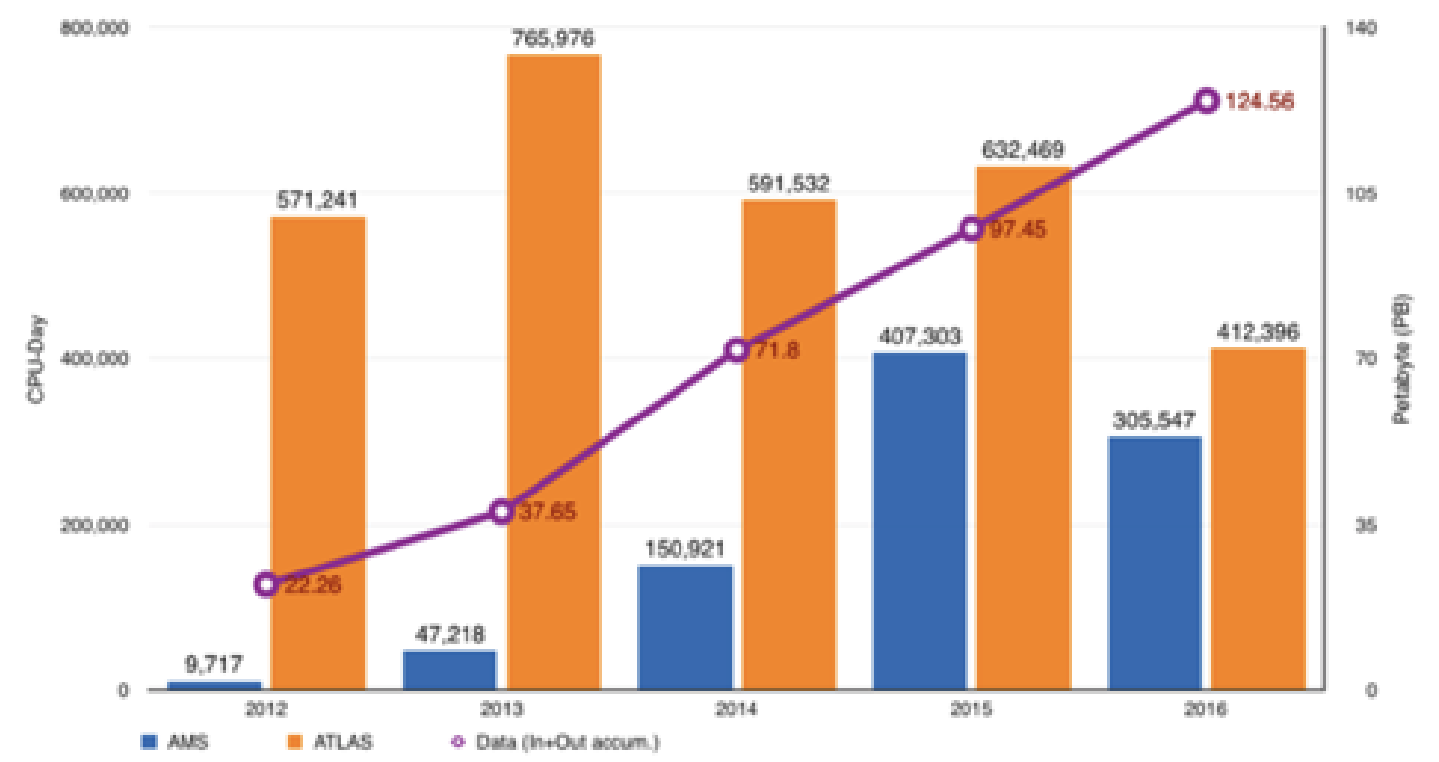

Figure 3.1. CPU Usage (in CPU-Day) of AMS computing on DiCOS 


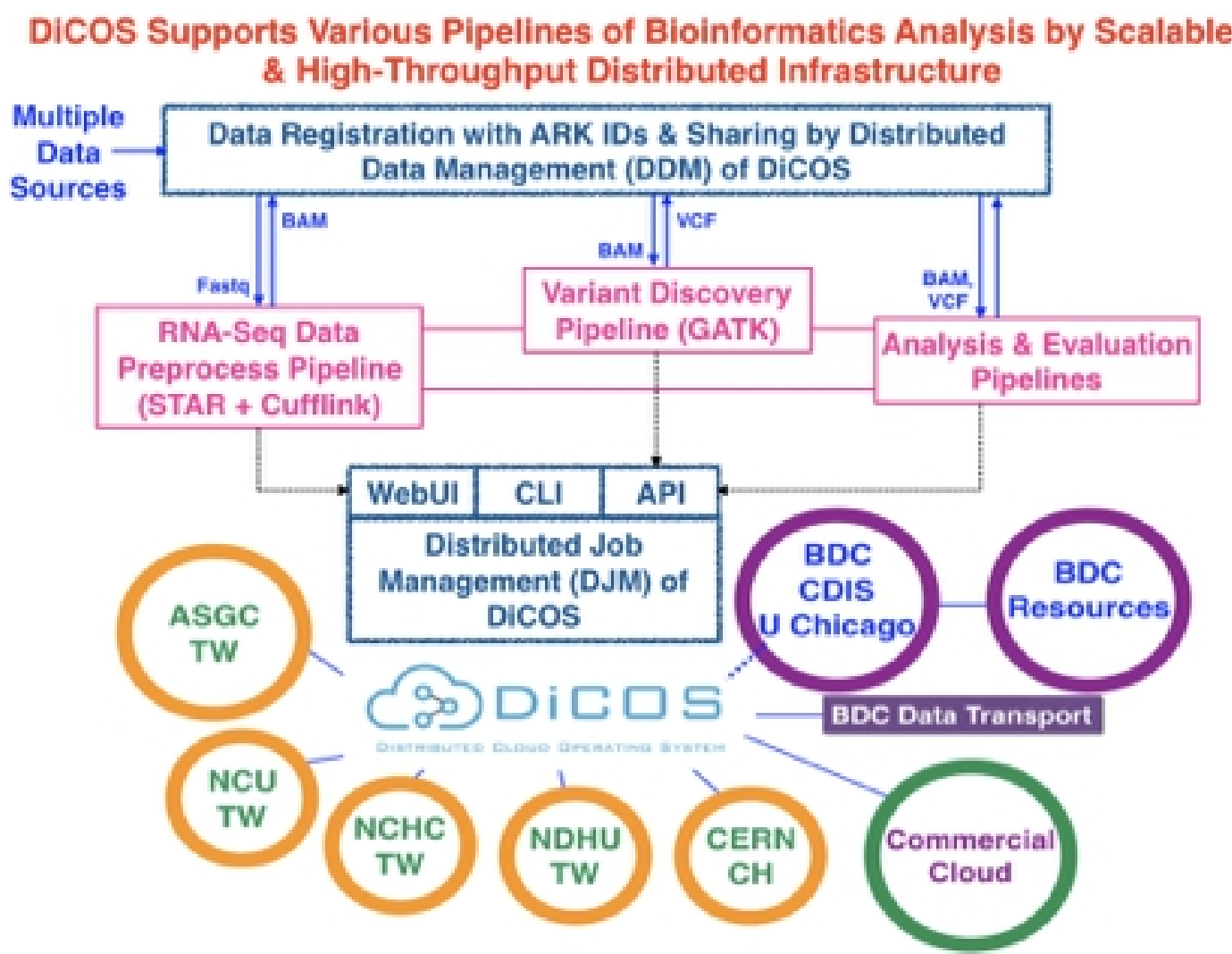

Figure 3.2. Bioinformatics Applications on DiCOS

23-Apr-2016 08:03:00 to 03-May-2016 08:01:00

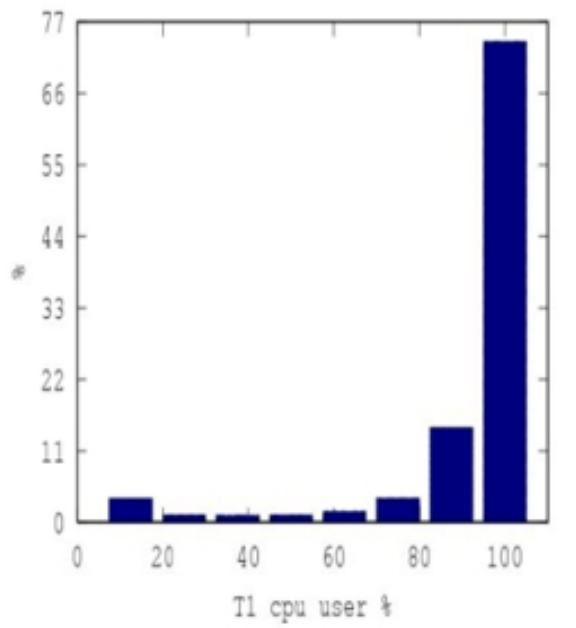

Single core jobs :

finithed dyration tine percentage : 89.99

failed diration tise sercestage : 6.12

cancelled derotion time percentage : 3.3

jobs efficiency : 0.8 pro

atage in tine percestage : 0.90

files transfer error time percentage : 1.89

witi-core jobs:

Piflesed datation tine perceatage : 76.72

talled datten tase percetcage : 21.03

cancelled darution time percentage : 0.19

joed efflelency : t...

flage in the percertage : $1.7 \%$

thea trater tror tine petcentaje : 17,61

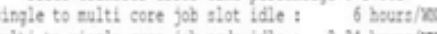

eulti to single core job sode idle: 3.34 hours/m

T1 cpu user t

Figure 3.3. Summarized jobs statistics of WLCG Tier-1 resources at ASGC 


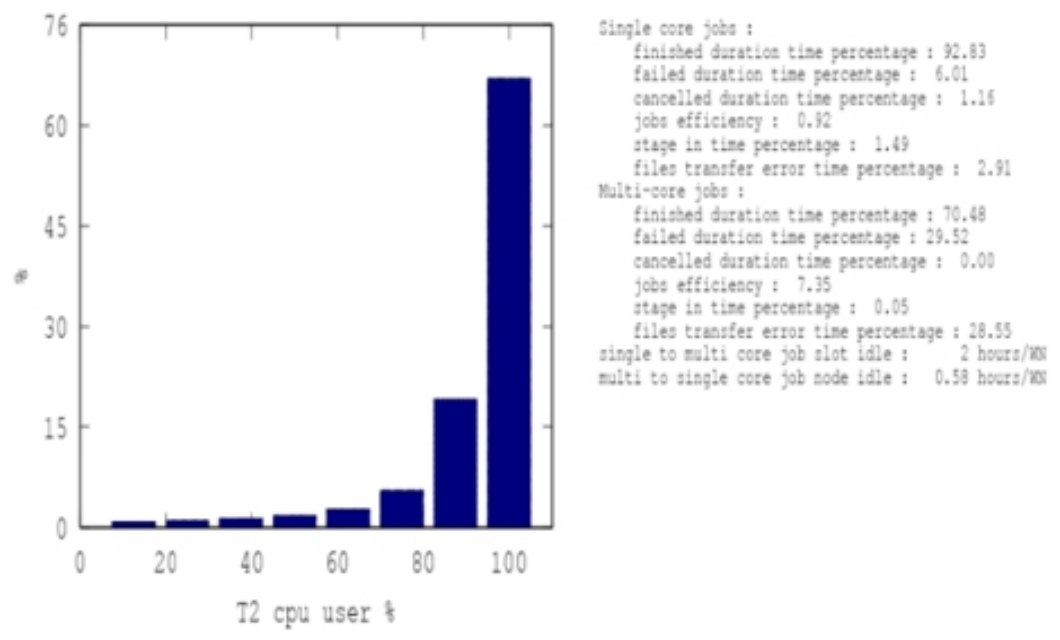

Figure 3.4. Summarized jobs statistics of WLCG Tier-2 resources at ASGC 